\title{
The Person at the Core of Psychological Science
}

\author{
JUAN F. FRANCK \\ Instituto de Filosofía, Universidad Austral, Argentina* \\ jfranck@austral.edu.ar \\ ORCID: 0000-0002-7480-0188
}

\begin{abstract}
The paper has been written from a philosophical perspective and triggered by the recurrent discussions in psychology about the most suitable methods to study our multifaceted subjectivity. Its main point is that a phenomenological understanding of the human person provides a robust and also flexible philosophical framework for psychology. The first part discusses three classical distinctions - individual/general; explaining/understanding; induction/interpretation- which, in spite of possible deficiencies, are useful to illustrate the specificity of the human sciences relative to the natural sciences. If not understood as an either-or dichotomy these distinctions represent the search of the right balance to reflect the complexity and richness of psychological science. The second part presents the phenomenological notions of 'vital reduction' and 'personalist reduction', where reductions does not take on an eliminativistic meaning, but of directing the mind's gaze to attend to what is originally the case. The 'vital reduction' reveals a subject of experience at the center of the lifeworld, and the 'personalist reduction' sees in rationality - i.e., the power to grasp the meaning of things and to recognize other subjects of experience - a deeper dimension of the subject, who we can thus call a person. Psycho-
\end{abstract}

I am particularly grateful to Erin Smith for her extremely helpful suggestions. Also to Scott Harrower, Ryan Peterson, Aaron James, and Juan Pablo Roldán for their very useful comments on previous drafts of this paper, and for the discussion at the TheoPsych Conference (Feb 3 and 5, 2021). This research was made possible through the support of an award from the TheoPsych project of Blueprint 1543. The opinions expressed are those of the author and do not necessarily reflect the views of Blueprint or the TheoPsych project. 
logy and phenomenology converge in disclosing the person-centeredness of our lifeworld.

Keywords: psychology; personalism; phenomenology; personalist reduction; humanistic psychology; hermeneutics.

The present paper has been written from a philosophical perspective and triggered by the recurrent discussions in psychology about the most suitable methods to study our multifaceted subjectivity. Its main point is that a phenomenological understanding of the human person provides a robust and also flexible philosophical framework for psychology. Its goal is not to suggest novel methods for psychology, nor is it to pursue a theoretical integration of multiple levels of analysis - cognitive, genetic, developmental, evolutionary, social, etc.- in the manner of an integrative pluralism (Kendler 2005; Lilienfeld 2007; Healy 2012). Its concern is more inter- or cross-disciplinary, than intra-disciplinary. Phenomenology as a philosophical school is a serious effort to be as faithful as possible to reality as given to our consciousness, and to bring to light the nature of consciousness itself. It has attracted psychology since its beginnings (Spiegelberg 1972), and phenomenological methods in qualitative psychology are a remarkable example of how psychology and phenomenology can work together (Englander 2016). This article, however, just calls for a more fruitful conceptual framework to make sense of the various methods in psychology.

The first part brings attention to three well-known pairs of notions: individual/general; explaining/understanding; induction/interpretation. In spite of their inadequacies, they are useful to illustrate the specificity of the human sciences relative to the natural sciences, or so I suggest. I then present a distinction within the phenomenological tradition that provides two significant bridge-concepts for a fruitful dialogue between philosophy and the scientific study of the human person: 'vital reduction' and 'personalist reduction' (Leocata 2007; 2010). As will become clear, the meaning of the term 'reduction' in phenomenology is opposite to any sort of eliminativism and may therefore appear unfortunate 
in this context. 'Reduction' in phenomenology aims at revealing a principle that informs and gives sense to our experience and behavior, without undercutting any other elements or dimensions involved. The sense of the 'vital reduction' is to reveal a subject of experience at the center of the lifeworld, of the world as it is lived. The 'personalist reduction' sees in rationality - i.e., the power to grasp the meaning of things and to recognize other subjects of experience - a deeper dimension in the subject. Psychology and phenomenology converge therefore in disclosing the person-centeredness of the world, an insight that is far from any form of subjectivism, as I hope will become clear in the second part.

\section{The methodological and theoretical richness of psychological science}

Although mental life is partially dependent on physical and biological phenomena, the study of subjectivity is not limited to the natural sciences. Neuroscientists, for example, assume an understanding of mental life and behavior to interpret electrograms and brain scans. These outputs do not provide them with criteria for interpreting that information but uncover some conditions and causes of the enactment, variations, and distortions of mental life and behavior. From a scientific perspective Krakauer et al. (2017) argue compellingly for behavioral research as a guiding vision for neuroscientific studies. The same applies to the formal sciences, increasingly engaged in the investigation of the natural world. It is one thing to say that complex nonlinear models are better suited to represent and formalize human behavior than more basic mathematical tools, and a very different one to claim that we would have thus achieved a sufficient explanation.

Relative to the neurosciences, psychology is much better placed as a scientific counterpart in a comprehensive study of the human person (Smith 2021). First, psychology deals with thoughts, beliefs and behavior without further justification; that is just its field. Second, psychology is also close to other kinds of empirical study, mainly to neurobiology and the social sciences. The social sciences, dealing with our cultural, social 
and intersubjective dimensions, are just as relevant as brain sciences to understanding mental life. Without yielding neither to neurobiological reductionism nor to social constructivism, however, the focus of psychology remains individual experience and subjectivity (Kirschner 2015).

In a recent contribution Danks and Eberhardt highlight the methodological strain in experimental psychology as a consequence of the pretense to be a natural science. They claim that psychological phenomena are relatively inaccessible, and mental states are not directly observable; hence we measure proxies, like behavior and reports. The authors observe that while it is accepted that individual differences make psychological data highly variable, the obvious fact that the data "come from intentional agents with their own desires, beliefs, etc." (Danks and Eberhardt 2019, 215) should be discussed more. Now, subjects themselves are often unaware of these differences. We therefore use various techniques to explore unconscious cognition, preferring behavioral to verbal measures, and this increases the interpretive load of psychological theories. Without yielding to pessimism, the authors soberly conclude that no experimental design is perfect.

Writing for the same volume Bechtel and Wright (2019) concentrate on two kinds of psychological explanation: nomological and mechanistic. What psychology often calls effects - the Garcia effect, the pratfall effect, etc.- relate stimuli with experiences and behavior, but remain at a descriptive level, leaving the question 'why?' unanswered. However, a lot of research today concentrates on what are called psychological mechanisms, trying to identify both the mental operations and the relevant brain parts involved in psychological phenomena. Mechanisms are represented as models and simulations with graphic displays, which provide a better understanding than propositional thinking or mathematical formulations and also help to account for individual cases, a central purpose of psychological science.

The two mentioned contributions prompt to reconsider psychology's peculiar position at a disciplinary crossroads and as the study of the person as a subject of experience. Both contribute to a greater awareness of the limitations of the methods used in the natural sciences for psychol- 
ogy, whose object is undoubtedly far more complex. The classical distinctions discussed below are not understood as an either-or dichotomy but represent the search of the right balance to reflect the complexity and richness of psychological science. Stressing one member of the distinction qualifies the extent and purpose of the other member and does not entail its rejection; roughly speaking, the reverse is true in the natural sciences. For a more extended treatment see (Leocata 2010, 167-203).

The first distinction is the renowned one between the individual and the general. It reminds of Wilhelm Windelband's description of the sciences of the spirit (Geisteswissenschaften), mainly historical sciences, as ideographic - literally: depicting the individual-, and of the natural sciences (Naturwissenschaften) as nomothetic - literally: establishing laws-. Influenced by contemporary psychological research carried out by figures such as Wundt, Weber and Fechner, Windelband himself placed psychology within the natural sciences, whereas history would represent the paradigm of a Geisteswissenchaft (Windelband 1894). However imprecise and disputable, such characterization reflects the prevalent focus at the time. The natural sciences study numerous individual objects (the planet Earth, the atmosphere, a pack of wolves, evolution, etc.), but they do so under the light of regularities obeyed in specific circumstances, revealed by observation and experience. Their individuality is still mostly an instantiation of a general pattern or set of laws. In turn, the sciences of the spirit also seek regularities, comparing cases and patterns of behavior or development in different individual persons and groups of people, but with the aim of making better sense of particular cases. Whereas exceptions and irregularities reveal insufficient information for the sake of formulating laws of nature, they are expected and regarded as customary in the human sciences, because the individual counts for them as such, in a way no natural scientist would admit. The study of the mechanisms of sense perception and other mental operations closely dependent upon physical properties is only a fraction of empirical psychology. We should therefore not expect the same kind of predictability in both kinds of sciences.

The second pair of notions is closely connected, and we owe it to another German philosopher, Wilhelm Dilthey, who used it with the same 
purpose as Windelband's. Following up on a distinction drawn by Johann Gustav Droysen, Dilthey did not focus on the kind of object, but on the kind of attitude scientists should approach their object with, and on the expected result in each kind of science. He proposed that the goal of the natural sciences is to explain (erklären), whereas human sciences seek to understand (verstehen), borrowing the latter term from the hermeneutical tradition starting from Schleiermacher (Von Wright 1971; Gantt and Williams 2016). In ordinary speech to explain and to understand are used almost as synonyms, but a finer analysis suggests a relevant nuance. To explain means to be able to identify the laws and causes that account for something, for its properties, reactions, etc., and even though determinism does not necessarily reign in the natural world, the verification that things with the same properties behave and react identically under the same circumstances, marks the path of progress for natural science. On the contrary, intentionality, motivation, and free will, without which we cannot make sense of the lived world of an individual person or of a group of people, are object of understanding. They are also causes, having visible effects in the world and in our behavior - often more than other causes- but they are causes of a different nature.

Just as the first distinction, this second one loses its relevance for the human sciences if understood as an either-or dichotomy. The why to which natural and human scientists seek an answer is different, but both look for regularities. Individuals and groups enjoy a reasonably large degree of autonomy, but they also tend to behave in partly predictable ways. Information about a wide variety of human situations, including past ones, and using quantitative methods and statistics, unquestionably contributes to a better knowledge of humanity. So, if explaining without understanding tends to ignore subjectivity, for an understanding blind to the search of regularities things can easily become arbitrary and ultimately also unintelligible. To put it in different terms, it is erroneous to see particular human situations as just examples of established laws, because some elements that need to be factored in the equation to account for them, such as motivation and intentionality, are only understandable as sense-making of those situations. 
The third distinction probably captures the specificity of the natural sciences more sharply. Since Bacon, Descartes and Galileo, modern science is built on induction as a process of generalization from observation and experience with the purpose of establishing laws, which describe the phenomena and open the way to new discoveries. Even though the natural sciences adopted induction first, there is no reason why it should not be useful for the human sciences as well. The difference between both kinds of sciences, therefore, lies not in induction as such but in how to understand the facts and terms involved.

In the human sciences establishing general tendencies and probabilities, from an inductive base as large as possible and from comparisons between cases, does not dispense with the interpretation of individual cases. Such an effort is necessary because actions and experiences acquire meaning within a personal context, where a perspective of the world is at play. Culture, beliefs, values, expectations, preferences are not simply facts that could be 'verified' like physical properties. And engaging with a constellation of meanings in order to capture a personal world requires an empathic relationship, which involves a double hermeneutic circle. On the one hand, each part or aspect of a person's world reflects to some extent the whole of that world and is rightly understood within it. So, the whole makes the parts more intelligible, and the parts clearly contribute to understanding the personal world as a whole. On the other hand, the unity of a world of meanings is given in and by each subject or person, who does not exist independently of that context. Although there always remains a certain ineffability to each person, understanding the context contributes to understanding the person, and vice versa.

The fruitful dialectic tension within these three methodological distinctions is reflected in the mutual complementarity of qualitative and quantitative methods in psychological science (Todd et al. 2004). Quantitative studies focus on one particular hypothesis, tested in the largest possible number of cases. Statistical methods and probabilities are used to arrive at general conclusions, which provide precise answers to specific questions. But they are not good at generating new hypotheses, nor do they provide any information regarding what it feels like to be in a given 
situation. The exploratory character of qualitative research recommends it instead for both purposes. By observing individuals and groups, or by conducting interviews with open-ended questions, it aims to capture the richness of human experience and to detect relevant nuances and differences, as well as commonalities. It provides descriptions of lived experience and behavior and is therefore able to suggest hypotheses for later quantitative research. The result is typically a narrative using labels to describe how subjects convey their experience and quoting their actual words when relevant. The narrative is mostly interpretative and takes recourse to a wide variety of theoretical elements. This circularity between data and theory, and the greater involvement of participants, convey qualitative research a highly dynamic character. Data gathered using qualitative methods can also be analyzed quantitatively, establishing recurrences, correlations, etc. Many researchers understandably prefer mixed methods, combining the advantages of both.

The methodology of psychological inquiry is multifaceted, because such is human psychology. The relatively simple regularities, or effects, and mechanisms that describe psychological phenomena, on the one hand, and the subtleties of individual interpretations of a person's world, on the other hand, suggest that a rich conceptual spectrum is necessary to make sense of our subjectivity. We are placed at multiple crossroads, and all of them are reflected in our psychology: we think, behave and act as embodied beings; we live embedded in a multi-layered social and cultural context; we have a more or less conscious worldview and uphold more or less strict ethical values. Biological and social factors are imbricated in our constitution, but without anchoring psychology in the individual person psychological claims may sound abstract or denaturalized. How are we to understand this interwovenness of all dimensions of the human person? In the following section I suggest some phenomenological clarifications that can contribute to delineating the intersection between philosophy and psychological science. 


\section{A theoretical contribution from phenomenology}

The term 'reduction' is definitely ambiguous in this context. A theory is said to be reducible when its laws can be understood as cases of more general laws. The project of naturalizing consciousness is based on this reductionist assumption insofar it rejects any explanation of consciousness that is not amenable to the natural or the formal sciences (Petitot et al. 1999). A stronger meaning of reduction is found in the eliminativistic claim that there is only one basic constituent of reality, most likely matter (Lombardi 2017).

In phenomenology, however, reduction has a completely different meaning and actually serves the opposite purpose. It means something like 'conducing', referring or directing the mind's gaze to attend to what is originally the case but probably not noticed in the first experience of an event. When speaking with somebody we pay attention to what is said, to the environment where we are having the conversation, to the span of time the conversation lasted, to the person we are talking to, etc.; in sum, we focus on the world and on what happens in the world. In Husserl's terms, ordinary life is carried on with a 'natural attitude' regarding things and people. But after that conversation we could adopt a different attitude and focus instead on our own experience: if we could see and hear the person well, what inner reactions we had during the conversation, whether it seemed brief or rather long to us, etc. All of this is no less certain than the previous consideration but was not necessarily apparent during the conversation. It reveals a new sphere of meaning, which is not perceived the same way as the 'objective' world. The world is given to us in and through that 'subjective' mediation, not as a brute fact but under some perspective, with some kind of participation of our own.

The scope of this different approach, the 'phenomenological attitude', ranges from the basic awareness that although we were listening to a person, listening is an act of ours - not what or whom we were listening toto realizing that we would have experienced the same conversation in a different way under other circumstances, had we been less tired, more relaxed or cheerful, for example, or had we entered the conversation with 
different expectations or purposes. This reduction discloses a range of acts, attitudes, moods, which are a condition for our involvement in and with the world. Husserl called it a phenomenological reduction, meaning that we are thus led - conduced- to discover the reality and the structure of conscious or mental life. Mental life has in fact a peculiar kind of consistency to itself and even an apodictic character, because it is immediately given. Although our attention is first directed to the world, the world appears to us unescapably through some subjective mediation, also partly depending on our bodily condition: things are given in space from a certain perspective; sensations depend upon our bodily constitution; the structure of time inextricably knits memory, attention and expectation; events are always lived and interpreted from some conceptual, biographical, cultural, ethical, and also philosophical background; actions answer to some motivation, etc.

No relativistic or idealist assumption is implied in recognizing that subjectivity shapes our experience of the world, but it would also be misleading to speak of all those dimensions as 'objective' in the naturalistic sense. They reveal the subject of experience, who is no less real than the world and is also required for the world to disclose its meaning. Husserl spoke of a "personalistic attitude, the attitude we are always in when we live with one another, talk to one another" (Husserl 1989, 192). The personalistic attitude, in which we recognize other persons, is in a way also natural, though not naturalistic, because it does not intend to see the world only through the eyes of the natural sciences, such as physics.

Our encounter with the world happens in many different ways but there are two particularly active levels: sensitivity and rationality. Both are distinguishable dimensions of human consciousness and conform the first-person perspective, i.e. how something appears to someone. Apart from sensory perception the first level includes emotions, feelings, and instincts. These already show a teleological relation to and with the world: emotions are answers to a given circumstance, but presuppose a directedness guiding and conditioning that response; feelings are also responses to reality and reveal at the same time the encountered world and how it affects the subject's own condition; instincts involve a first degree of 
instrumentalization of reality according to some subjective interest. So, sensitivity already configures a world of meanings, where passivity and activity are deeply interweaved.

This account of sensitivity presents some similarities with the enactive approach to the cognitive sciences (Thompson 2007). Enactivism postulates a continuity between life and mind, emphasizing the active character of living organisms in the configuration of their environment. It characterizes living organisms as sense making. A world of meaning begins to exist with life, because things can go either right or wrong for a life form, even in the absence of consciousness. The beneficial and the harmful are actively defined by the organism relatively to its own survival, and the same goes for the environment (Umwelt) of an animal. Moreover, an environment would not exist as such without reference to a living system: it is the world (Welt) surrounding $(u m)$ an organism. For enactivism phenomenal or conscious life presents a similar kind of subject-centeredness, characterized by a higher degree of subjective configuration of the world. Enactivists rightly call attention to the existence of some kind of inwardness even at the most basic levels of life, as well as to the primordial character of activity. But they also tend to reduce all sense making to a more or less complex process of what it calls structural coupling between an organism and the environment. It does not regard consciousness as a clear break with more basic phenomena, but only as a more complex manifestation of the same kind of processes that already guide the self-organizing of cells.

Enactivism is part of a more general departure from classic cognitivism and representational theories of the mind that endorses an embodied cognition-extended mind approach to psychological explanation (Adams and Aizawa 2019). The departure from cognitivism takes on different forms, from simply playing down the role of representations in cognition to radical forms claiming that the mind is just an adaptation for coping with the environment (Hutto and Myin 2012). These radical forms appeal to different theoretical resources: dynamical systems theory, the sensorimotor theory of perception, or an extended theory of mind. As Adams and Aizawa point out, none of them is free of difficulties. 
Dynamical systems theories can explain how some basic stimulusdriven behaviors, such as feeding, can be performed without mental representations. The authors observe, however, that the fact that a Venus flytrap can obtain food does not prove that cognition can take place without any mental representation, but rather that "the task of obtaining food simply does not require cognition" (Adams and Aizawa 2019, 203). The sensorimotor theory of perception is close to enactivism (Noë 2006) and claims that action is constitutive of perception. But both ordinary experience and simple experiments showing that sense perception does not disappear in the absence of action or movement, seriously question the viability of any radical form of this approach. Few would deny also that there is a relation of complementarity between brain processes, the body in action and the social environment, but this is hardly evidence that such a structured system is the equivalent of a cognitive being, as proposed by theorists of the extended mind (Clark and Chalmers 1998; Clark 2008). If cognition is defined by coupling, there would also be cognition in a thermostat. In spite of all its shortcomings, however, the approach supports the idea that cognition and behavior are not understandable as acts of a disembodied and isolated subject, and that it is misguided to speak without qualifications of an 'inner' mind and an 'outer' world. Although its radical claims are theoretically interesting, the approach is attractive because it can assume mild claims that almost nobody would reject (Adams and Aizawa 2019).

This digression about enactivism and the embodied cognition-extended mind approach proves useful to highlight an important distinction. Even though they presuppose the surrounding objects, life and feelings are essentially self-centered and configure an environment that is relative to that center. All their activity tends to conserve, defend and expand life. In contrast, although also rooted in a subject, rationality is defined by the opposite direction: its purpose is to come to grips with the objective truth about reality. It reveals a more intense form of intentional life, and also a richer engagement with the world, opening up a whole new level of activities for the subject: culture, science, ethical behavior, etc. The world is infinitely enlarged by rationality not by reducing anything to a subject's 
needs and convenience, but by knowing and acting according to the objective nature of things. Thanks to reason we exist facing up to the whole universe. Josef Pieper expressed this idea by saying that while animals have an environment (Umwelt, surrounding world), the human being has a world (Welt), meaning the whole of reality (Pieper 1998, 99ff.).

The apparently trivial gain of rationality enables objective knowledge, helps redirect potentially negative impulses and urges to more constructive aims, makes ethical behavior, and the ensuing responsibility, possible, and so on. Although it can be put to serve the subject's needs, the proper horizon of rationality is being in all its universality, moving far beyond any approach centered around basic forms of life.

There are therefore a centripetal and a centrifugal intentionality. Although both are present at the level of emotions, where centripetal affectivity co-exists with centrifugal behavior (Fuchs 2020, 22), the distinction assumes a higher relevance when contrasting the realm of feelings with that of reason. In the former objects largely gravitate around the subject, while in the latter the subject expands its range potentially to the whole of reality. Reason is directed towards the objective nature and value of things, and to others as others; it is not first and foremost concerned with how the subject is affected by them. Even the person's own existence and place in the world can only be grasped from the rational perspective, since rationality allows to conceptualize a world and recognize something as having a meaning. The ability to relate to other persons as autonomous agents, possessing their own subjectivity, also goes hand in hand with rationality, because interpersonal relationships presuppose acknowledgment of the other as a different center of the world, not as existing only within my own world (Zahavi 2014). And such an attitude is obviously at the core of any ethical behavior as well. However, a certain person-centeredness remains also in centrifugal intentionality proper to rationality, because the possibilities of realization for the person increase with it.

Phenomenologically speaking, the disclosure of subjectivity is therefore given in a twofold layered reduction. A first layer is what we may call, following Francisco Leocata, a 'vital reduction', which consists in "letting human experiences [vivencias; Erlebnisse in German] come forward in all 
their breadth, according to the perspective and methodology of each human science" (Leocata 2010, 164). Here we simply acknowledge experience as it is given, revealing a subject who engages in the lifeworld with other persons in a profuse variety of ways, studied by different human sciences (history, psychology, aesthetics, linguistics, etc.). As we have seen that lifeworld is already pervaded by subjectivity, and therefore reveals and reflects our human experience of the world (Leocata 2010, 17).

Although not always recognized, a second layer lies deeper at the root of this vital reduction, informing it from within and conveying it a more profound meaning. We do not live like other creatures but transcend the immediacy of the lifeworld, asking ourselves about the nature of things and about their relative value. This radically novel way to address the world is manifested not only in pragmatic behavior, but also and mainly in the works of culture, which embody an understanding of nature and of ourselves. Without suppressing or ignoring the pragmatic domain and our immediate interests, the subjectivity that pervades the human lifeworld goes far beyond them, because we exist facing the totality of being. The human lifeworld is not simply the natural world, but a cultural and social world; both represent two different levels of the same personal life having nonetheless a partially independent dynamic. Besides, it can also bear signs of our need to transcend it and of a deeper questioning about the nature of things. The vital reduction reveals thus at its core what Leocata calls a 'personalist reduction', because our world is the lifeworld of a person, facing the totality of being and existing together with other persons (Leocata 2007, 167).

It is important to see the vital and the personalist reductions as part of one same reduction: our embodied make-up and our social existence bear a spiritual and personal meaning. All human sciences assume a world of meanings and values that gives sense to their own object. But that world is not fully understandable from the perspective of the human sciences without philosophical, ethical, and sometimes also theological clarifications. The aim of the reduction is not to uncover something that would exist separately or with complete independence of everyday experience, a sort of ego or disembodied subject that would only come to light after 
removing everything that tends to be confused with it. Husserl acknowledged that his own early analyses tended to present the 'I' or ego as "empty of content", therefore suggesting that nothing had been won (Crisis $\S 43$; Husserl 1970, 155). But that would be a caricature of phenomenology. There is no split between the person and the lifeworld, and the aim of the reduction is to reveal the fullness of experience, going beyond a superficial look that contents itself with the first appearances. Its purpose is not to have us withdraw from ordinary life, but to live in a richer way, aware of the deeper layers of existence.

\section{Conclusive remarks}

It would be interesting to contrast the present proposal of paying attention to phenomenology in our understanding of the person with William Stern's critical personalism (Stern 2010; Lamiell 2003; Lehmann-Muriithi et al. 2016). Stern stands between what he calls a naïve personalism, which places an independent I as the source of unity in the person, and impersonalism, which treats the person as a thing, a by-product of mechanisms. His understanding of the person as a unitas multiplex integrating different kinds of teleologies is a very fruitful notion. Discursive psychology, based on Wittgensteinian semantics, is also another recent trend in psychology that attempts to do justice to a larger picture of the human being (Harré 1998). In all three cases, what is at stake is the capacity to provide a framework that integrates various methodologies and confers a comprehensive and coherent understanding of all aspects of personhood: biological, mental, cultural, ethical, religious, etc.

I have used the term 'person' somewhat loosely in the previous pages. I am aware that just as on almost anything, there is hardly any agreement in philosophy on what a person is. And the same may apply to psychological science. Without pretending to settle any discussion, however, I would like to propose five statements that characterize a personalist approach as I see it:

a. A person is an individual subject of experience and agency, i.e., of passivity and activity. 
b. The body essentially qualifies the person's life, providing the means for the person's being-in-the-world and a fundamental source of growth and development.

c. Rationality is also essential to persons, opening them to truth, culture, and values.

d. Both embodied nature and rationality make the person's social being possible.

e. A person's full flourishing requires relatedness to other persons, so that a totally isolated person would be impossible to conceive.

Even though these claims go beyond a mere phenomenology of the person, they are grounded in a phenomenological reading of human existence (Zahavi 2003).

To some the claim that the person is at the core of psychological research may sound outlandish. Maybe the mind or the self, but 'person' looks too theoretical a notion. To others, on the contrary, it may sound like a platitude. What else would psychology deal with? Nonetheless, the repeated attempts to interpret the human psyche as a result of biochemical or sociocultural processes, and the complexity of human experience and behavior, suggest that it is anything but trivial to revise once and again the philosophical framework from which we interpret the results of psychological research. Besides, systematically confronting the results of research with philosophical knowledge contributes to a greater awareness of some presuppositions in psychological theory that may otherwise not easily come to light. That framework is also reflected in the methodology employed and influences both research and theory.

My contribution neither intended to propose a specific methodology nor another psychological theory or model. I wanted to suggest, first, that the different methodological approaches make more sense if interpreted from an un-prejudiced philosophy of the human person. Second, that the concept of person can serve as an anchor for different levels of information and meaning - physiological, behavioral, phenomenal, cultural, social, ethical, etc.- and possibly also help achieve an integration of those levels. With the metaphor of an anchor, I mean a central core that keeps a plurality of dimensions bound together in such a way that these not 
only qualify that same central core but are also united and integrated among themselves. The import of each dimension and their relative value derive from their affecting the lifeworld of a person. The phenomenological reduction has led to the discovery of a core irreducible to any single dimension, but at the same time able to integrate them all.

\section{References}

Adams, Fred and Aizawa, Ken. 2019. "Embodied cognition and the extended mind." In The Routledge companion to philosophy of psychology, edited by S. Robins, J. Symons and P. Calvo, 193-213. London and New York: Routledge. Bechtel, William and Wright, Cory D. 2019. "What is psychological explanation?”. In The Routledge companion to philosophy of psychology, edited by S. Robins, J. Symons and P. Calvo, 113-130. London and New York: Routledge.

Clark, Andy (ed.). 2008. Supersizing the mind. Embodiment, action, and cognitive extension. Oxford: Oxford University Press.

Clark, Andy and Chalmers, David. 1998. "The extended mind." Analysis 58 (1): 7-19.

Danks, David and Eberhardt, Frederick. 2019. "Conceptual problems in statistics, testing and experimentation." In The Routledge companion to philosophy of psychology, edited by S. Robins, J. Symons and P. Calvo, 214-230. London and New York: Routledge.

Englander, Magnus. 2016. "The phenomenological method in qualitative psychology and psychiatry." International Journal of Qualitative Studies on Health and Well-being (11): 1, 30682.

Fuchs Thomas. 2020. “Verkörperte Emotionen und ihre Regulation.” In Handbuch Emotionsregulation, edited by S. Barnow. Berlin and Heidelberg: Springer. DOI: 10.1007/978-3-662-60280-5_2.

Gantt, Edwin E. and Williams, Richard N. 2016. "Explanation versus understanding in psychology: A human science approach." In The qualitative vision for psychology: An invitation to a human science approach, edited by C.T. Fischer, L. Laubscher and R. Brooke, 31-48. Pittsburgh: Duquesne University Press.

Harré, Rom. 1998. The Singular Self. An Introduction to the Psychology of Personhood. London: Sage.

Healy, Paul. 2012. "Toward an integrative, pluralistic psychology: On the hermeneuticodialogical conditions of the possibility for overcoming fragmentation.” New Ideas in Psychology (30): 271-280. 
Husserl, Edmund. 1970. The crisis of European sciences and transcendental phenomenology, translated by David Carr. Evanston: Northwestern University Press.

Husserl, Edmund. 1989. Ideas pertaining to a pure phenomenology and to a phenomenological philosophy. Second book: Studies in the phenomenology of constitution, translated by R. Rijcewicz and A. Schuwer. Dordrecht-Boston-London: Kluwer.

Hutto, Daniel and Myin, Erik. 2012. Radicalizing enactivism. Basic Minds without Content. Cambridge, MA: MIT Press.

Kendler, Kenneth S. 2005. "Toward a philosophical structure for psychiatry." American Journal of Psychiatry (162):433-440. DOI: 10.1176/appi.ajp.162.3.433.

Kirschner, Suzanne R. 2015. "Subjectivity as sociocultural constituted experience." In The Wiley handbook of theoretical and philosophical psychology, edited by J. Martin, J. Sugarman and K.L. Stanley, 293-307. Oxford: Willy Blackwell.

Krakauer, John W., Ghazanfar, Asif A., Gomez-Marin, Alex, MacIver, Malcolm A. and Poeppel, David. 2017. "Neuroscience Needs Behavior: Correcting a Reductionist Bias.” Neuron 93 (3): 480-490. DOI: 10.1016/j.neuron.2016.12.041.

Lamiell, James T. 2003. Beyond individual and group differences: Human individuality, scientific psychology, and William Stern's critical personalism. London: Sage.

Lehmann-Muriithi, Kolja, de Resende Damas Cardoso, Carolina and Lamiell, James T. 2016. "Understanding Human Being Within the Framework of William Stern's Critical Personalism: Teleology, Holism, and Valuation.” In Psychology as the science of human being. The Yokohama Manifesto, edited by J. Valsiner et al., 209-223. Springer: Switzerland.

Leocata, Francisco. 2007. Estudios sobre fenomenología de la praxis. Buenos Aires: Proyecto.

Leocata, Francisco. 2010. Filosofía y ciencias humanas. Hacia un nuevo diálogo interdisciplinario. Buenos Aires: Educa.

Lilienfeld, Scott O. 2007. “Cognitive Neuroscience and Depression: Legitimate Versus Illegitimate Reductionism and Five Challenges." Cognitive Therapy and Research (31): 263-272. DOI: 10.1007/s10608-007-9127-0.

Lombardi, Agustina. 2017. "Dan Zahavi and John Searle on Consciousness and Non-Reductive Materialism”. Scientia et Fides 5 (2): 155-170. DOI: 10.12775/ SetF.2017.020.

Noë, Alva. 2006. Action in perception. Boston, MA: MIT Press. 
Petitot, Jean, Varela, Francisco José, Pachoud, Bernard and Roy, Jean-Michel (eds.). 1999. Naturalizing phenomenology. Issues in contemporary phenomenology and cognitive science. Stanford, CA: Stanford University Press.

Pieper, Josef. 1998. Leisure. The basis of culture. South Bend, IN: St. Augustine's Press.

Smith, Erin I. 2021. "A Tale of Two Perspectives: How Psychology and Neuroscience Contribute to Understanding Personhood.” Scientia et Fides 9 (2): 35-53. DOI: 10.12775/SetF.2021.017.

Spiegelberg, Herbert. 1972. Phenomenology in psychology and psychiatry: A historical introduction. Evanston, IL: Northwestern University Press.

Stern, William. 2010 [1917]. "Psychology and personalism (trans. J.R. Lamiell)." New Ideas In Psychology 28: 110-134.

Thompson, Evan. 2007. Mind in life. Cambridge, MA: Belknap Harvard.

Todd, Zazie, Nerlich, Brigitte, McKeown, Suzanne and Clarke, David D. 2004. Mixing methods in psychology: The integration of qualitative and quantitative methods in theory and practice. London, UK: Psychology Press.

Von Wright, Georg Henrik. 1971. Explanation and understanding. Routledge \& Kegan Paul: London.

Windelband, Wilhelm. 1894. "Geschichte und Naturwissenschaft.” Translated by Guy Oakes as "History and Natural Science”, History and Theory 1980, 19 (2): 169-185.

Zahavi, Dan. 2003. Hussserl's Phenomenology. Stanford, CA: Stanford University Press.

Zahavi, Dan. 2004. Self and other: exploring subjectivity, empathy and shame. Oxford: OUP. 